\title{
Hétérotopie gliale pulmonaire : une lésion exceptionnelle chez un nourrisson avec un jumeau anencéphale
}

\section{Pulmonary glial heterotopia : unique lesion in an infant with anencephalic twin}

Tournier Louis (1), Berrebi Dominique (2), Peuchmaur Michel (2), Bonnard Arnaud (3), Belarbi Nadia (4), Sibony Olivier (5), Morcrette Guillaume (2)

(1) Service de pathologie, hôpital universitaire Robert-Debré, AP-HP, 48, boulevard Sérurier, 75019 Paris, France. Université Diderot Paris 7 - SPC, 16, rue Huchard, 75018 Paris, France.

(2) Service de pathologie, hôpital universitaire Robert-Debré, AP-HP, 48, boulevard Sérurier, 75019 Paris, France. Université Diderot Paris 7 - SPC, 16, rue Huchard, 75018 Paris, France.

(3) Service de chirurgie générale, hôpital universitaire Robert-Debré, AP-HP, 48, boulevard Sérurier, 75019 Paris, France. Université Diderot Paris 7 - SPC, 16, rue Huchard, 75018 Paris, France

(4) Service de radiologie, hôpital universitaire Robert-Debré, AP-HP, 48, boulevard Sérurier, 75019 Paris, France. Université Diderot Paris 7 - SPC, 16, rue Huchard, 75018 Paris, France

(5) Service de gynécologie obstétrique, hôpital universitaire Robert-Debré, AP-HP, 48, boulevard Sérurier, 75019 Paris, France. Université Diderot Paris 7 - SPC, 16, rue Huchard, 75018 Paris, France

Auteur pour correspondance :

Dominique Berrebi (2) ; Adresse électronique : dominique.berrebi2@aphp.fr 


\title{
Résumé :
}

L'hétérotopie pulmonaire gliale est une entité extrêmement rare dont la physiopathologie demeure incertaine. Nous rapportons pour la première fois un cas survenu chez un bébé de 1 mois, indemne de toute malformation. Elle est issue d'une grossesse gémellaire monochoriale monozygote où l'autre jumelle atteinte d'anencéphalie exencéphalie a bénéficié d'une interruption médicale de grossesse. La maladie s'est manifestée par une détresse respiratoire transitoire néonatale, récidivante à un mois de vie, avec découverte sur la radiographie thoracique d'images kystiques pulmonaires plus marquées à droite. Devant la suspicion de malformation adénomatoïde kystique pulmonaire (MAKP), elle a été opérée à l'âge de quatre mois d'une lobectomie supérieure et inférieure droite. L'étude anatomopathologique retrouvait une lésion multi-kystique dont les parois étaient constituées de tissu glial bien différencié et peu cellulaire, par endroit, bordé d'épithélium bronchique. Il n'y avait pas d'argument morphologique en faveur d'une MAKP. L'évolution a été favorable avec un recul de cinq mois après l'intervention. Il s'agit d'un des très rares cas où la maladie n'a pas conduit à un décès rapide in utéro ou en périnatal immédiat. Cela suggère qu'une prise en charge efficace par chirurgie pourrait être un élément décisif dans la survie de ces patients.

\section{Mots clés :}

Hétérotopie gliale pulmonaire ; malformation adénomatoïde kystique ; détresse respiratoire ; anomalie du tube neural ; anencéphalie ; jumeaux.

\begin{abstract}
:
Pulmonary glial heterotopia is an extremely rare entity whose pathophysiology remains unclear. We report for the first time one case of pulmonary glial heterotopia occured in a one month baby free from any malformation. She has the particularity of being born from monozygotic monochorionic twin pregnancy where her anencephalic exencephalic sister benefited from a medical termination of pregnancy. She presented neonatal respiratory distress which recurred one month later. Chest X-ray revealed bilateral cystic pulmonary lesions mainly located in the right lung. Given the suspicion of congenital cystic adenomatoid malformation (CCAM), she underwent an upper and a lower right lung lobectomy at four months-old. The pathological study found a multi-cystic lesion consisted of welldifferentiated and poorly cellular glial tissue sometimes lined by bronchic epithelium. There was no pathological evidence for a CCAM. The evolution was favorable after surgery with an infant who was well five months later. This is one of the very few cases where the disease did not lead to rapid death in utero or during the perinatal period. This suggests that effective management by surgery could be a decisive factor in the survival of these patients.
\end{abstract}

\section{Key words :}

Pulmonary glial heterotopia; Congenital Cystic Adenomatoid Malformation; respiratory distress; neural tube defects; anencephaly; twin.

\footnotetext{
Abréviations :

MAKP : Malformation adénomatoïde kystique pulmonaire

SA : Semaines d'aménorrhée
} 


\section{$\underline{\text { Introduction : }}$}

La présence de tissu glial hétérotopique au niveau des poumons demeure extrêmement rare avec moins de 25 cas décrits (1). Si la majorité des cas publiés concerne des nouveaux-nés avec des anomalies cranio-faciales ou plus fréquemment du tube neural à type d'anencéphalie (2), elle survient de manière exceptionnelle chez les nouveaux-nés bien portants. L'évolution est le plus souvent fatale, soit in utero, soit en période néonatale. Nous rapportons le premier cas d'hétérotopie gliale kystique pulmonaire découverte après une grossesse gémellaire monochoriale monozygote chez un nourrisson (jumelle 2) sans aucune autre anomalie associée, opérée avec succès à l'âge de 4 mois.

\section{Histoire clinique :}

La Jumelle 2 était née d'une mère de 24 ans, primipare dont le principal antécédent était une mutation ARG506 du facteur V hétérozygote. Le père n'avait pas d'antécédent particulier. Il a été porté le diagnostic d'acranie-exencéphalie chez la jumelle 1 lors du dépistage échographique du premier trimestre. Il a alors été décidé de réaliser une interruption sélective de grossesse à 18 semaines d'aménorrhée (SA).

Les suites de la grossesse pour la jumelle 2 ont été marquées par une rupture prématurée des membranes à $20 \mathrm{SA}$ avec anamnios. Le suivi échographique n'a pas retrouvé d'anomalie morphologique. Il a été réalisé une IRM anténatale à $31 \mathrm{SA}$ et 1 jour, destinée à explorer le cerveau de la jumelle 2, qui retrouvait uniquement une dolichocéphalie. L'accouchement a eu lieu par voie basse sous anesthésie péridurale. La jumelle 2 était en siège décomplété lors de ce dernier. A la naissance, la jumelle 2 pesait $2110 \mathrm{~g}$ ( $22^{\text {ème }}$ percentile), mesurait $43 \mathrm{~cm}$ (14 ${ }^{\text {ème }}$ percentile), avait un périmètre crânien à $32 \mathrm{~cm}$ (49 ${ }^{\text {ème }}$ percentile) et un score d'APGAR évalué à 9/10/10.

Dans les suites immédiates de l'accouchement, la jumelle 2 a présenté une hypoglycémie, une discrète anémie, un ictère modéré ainsi qu'un épisode de détresse respiratoire résolutif ayant nécessité une ventilation en pression partielle continue à $+5 \mathrm{mmHg}$ et $21 \%$ de $\mathrm{FiO} 2$ pendant 1 heure. L'examen clinique retrouvait une monoparésie du membre supérieur gauche et un doute sur un pied creux à droite pour lesquels a été réalisée une échographie des hanches et de la moelle dans les premiers mois du post partum. L'échographie des hanches était sans particularité. L'échographie médullaire retrouvait une image linéaire hyperéchogène au niveau du cône terminal, évocatrice de lipome, dont la présence a été infirmée par l'IRM médullaire. En parallèle à ces explorations, la jumelle 2, après un intervalle libre de 1 mois, a de nouveau présenté une détresse respiratoire se manifestant par une polypnée persistante et des épisodes de cyanose péribuccale lors des périodes d'agitation. Il a été réalisé un bilan d'imagerie thoracique par radiographie (figure 1A) puis un scanner injecté (figure 1B et 1C). L'examen tomodensitométrique retrouvait au niveau du parenchyme pulmonaire de multiples lésions kystiques, bilatérales, multiloculaires et de taille variable prédominant très nettement au niveau du poumon droit et refoulant le médiastin vers la gauche. Des lésions parenchymateuses condensantes nodulaires et de petits troubles ventilatoires à type d'atélectasie étaient associés. Le premier diagnostic évoqué à l'issue de ces examens était celui de malformation adénomatoïde kystique. Après relecture de l'IRM anténatale réalisée à 31 SA et 1 jour, il existait déjà des lésions hétérogènes en discret hyposignal $\mathrm{T} 2$ et prédominant nettement à droite (figure 1D). A la suite d'une discussion multidisciplinaire, il a 
été décidé de réaliser une résection chirurgicale des lobes pulmonaires supérieur et inférieur droit.

\section{Examen pathologique :}

La pièce de lobectomie supérieure droite pesait $10,2 \mathrm{~g}$ et mesurait $8 \times 3 \mathrm{~cm}$ et celle de lobectomie inférieure droite $14,9 \mathrm{~g}$ et $5,5 \times 5 \mathrm{~cm}$. L'analyse macroscopique des lésions retrouvait plusieurs lésions kystiques intra-parenchymateuses dont les plus volumineuses mesuraient 4,5 x 4,5 cm et 5,5 x $5 \mathrm{~cm}$ (figure 2). Il n'y avait pas de végétation intrakystique. Sur certains kystes on retrouvait un épaississement blanchâtre des parois.

Histologiquement, il s'agissait de lésions kystiques disséminées au sein du parenchyme pulmonaire (figure 3A). La paroi des kystes était constituée de tissu glial bien différencié et peu cellulaire agencé en foyers vaguement nodulaires (figure $3 \mathrm{~B}$ ) avec, en surface, la persistance d'un épithélium bronchique (figure 3C). Il n'y avait pas d'autre tissu et notamment pas de muscle strié, de cartilage, de plexus choroïde, de tissu adipeux, d'annexe pilo-sébacée ou de kyste à revêtement cutané. On observait quelques structures bronchiolaires résiduelles au sein du tissu glial. Il n'y avait pas d'embole vasculaire de tissu glial. A distance des kystes, le parenchyme pulmonaire était normal.

L'étude immunohistochimique retrouvait une expression cytoplamique hétérogène de la PS100 par le tissu glial (figure 3D). Par ailleurs, il n'y avait pas d'expression de l'alpha-foetoprotéine, de l'hormone chorionique gonadotrope humaine, ni de Phox $2 \mathrm{~b}$. Le diagnostic final retenu était celui d'hétérotopie gliale pure kystique pulmonaire multifocale.

Les suites opératoires ont été simples avec une patiente redevenue eupnéique et une bonne évolution clinique lors de la consultation de suivi à cinq mois.

\section{$\underline{\text { Discussion : }}$}

Nous reportons ainsi le cas d'une hétérotopie gliale pulmonaire découverte chez une jumelle issue d'une grossesse monochoriale monozygote dont la sœur présentait une anencéphalieexencéphalie. Les localisations ectopiques de tissu neuroglial sans défect sévère du système nerveux central sont bien connues au niveau du nez («gliome nasal »), de l'orbite, du palais, de la peau et de la région sous-mandibulaire. Cependant, la présence de tissu glial bien différencié au sein de lésions pulmonaires kystiques reste exceptionnelle. Dans les très rares cas publiés d'hétérotopie gliale sans anomalie cranio-faciale ou du tube neural, il a été décrit d'autres lésions associées tel qu'un pancréas ectopique (1) ou un tératome (2). Dans notre cas, il a été suspecté un lipome du cône terminal qui a été ensuite infirmé sans que les imageries complémentaires ne décèlent une autre anomalie. Un seul cas décrit ressemble à celui que nous rapportons (1) avec un bébé issu d'une grossesse gémellaire avec une jumelle anencéphale décédée deux heures après la naissance. La deuxième jumelle a présenté à $\mathrm{J} 20$ une détresse respiratoire avec à l'imagerie de multiples lésions kystiques dans les deux champs pulmonaires, de taille moyenne à modérée, évoquant comme dans notre cas une MAKP. La patiente est décédée comme la majorité des cas rapportés dans la littérature $(1,3-$ 5). Le principal diagnostic différentiel est la MAKP tant au point de vue clinique qu' au point de vue histologique, d'autant qu'il a été rapporté l'association d'une hétérotopie neurogliale et d'une MAKP de type II (6). Histologiquement, on ne retrouvait pas dans notre cas l'aspect 
typique de MAKP de type II qui habituellement se compose de multiples kystes bordés par un épithélium cuboïdal ou cylindrique cilié de type bronchiole-like associé à du tissu musculaire lisse. De plus, la bilatéralité des lésions (très rares dans les MAKP) (7), le contexte clinique avec les notions de grossesse gémellaire et d'anomalies cranio-faciales chez l'autre jumelle, plaident en faveur d'une hétérotopie gliale pulmonaire.

Les hypothèses étiopathogéniques restent à ce jour très discutées (8). L'une d'entre elles serait une anomalie de la différenciation ou de la migration des cellules de la crête neurale. Cette théorie est appuyée par les anomalies cranio-faciales fréquemment présentes (2) et la description d'un cas d'hétérotopie gliale associée à une anémie de Fanconi avec une anomalie de la protéine FANCA qui joue un rôle important dans le développement du cerveau (9). Certaines des publications mentionnent, soit une hétérotopie gliale $(1,2,7,9)$, soit une hétérotopie neurogliale pulmonaire $(3,4)$. La deuxième hypothèse, moins vraisemblable, est l'aspiration de tissu cérébral fœtal lors de défects du tube neural avec l'implantation de fragments de tissu cérébral aspirés à partir du liquide amniotique chez l'autre jumeau (1). Le caractère bilatéral des lésions et l'absence d'argument histopathologique en faveur d'une aspiration ne plaident pas pour cette hypothèse. De plus la majorité des autopsies pratiquées pour anencéphalie ne retrouvent pas d'hétérotopie gliale pulmonaire (10). Une autre hypothèse est l'embolisation de tissu cérébral après un accouchement difficile par voie vaginale avec utilisation de forceps ; celle-ci est improbable puisque la majorité des lésions observées se situent dans les bronches et non dans les vaisseaux (2). Enfin, l'hypothèse d'une lésion hamartomateuse ou tératomateuse a été soulevée. Le caractère glial ou neuroglial pur sans aucun autre tissu tératomateux, le mode diffus des kystes au sein du parenchyme pulmonaire et le caractère bilatéral des lésions pulmonaires ne sont pas en faveur de ces diagnostics (7).

Du point de vue du traitement, la chirurgie par lobectomie pulmonaire du côté le plus atteint a contribué à l'amélioration de l'état respiratoire de la jumelle 2 et surtout de sa survie même s'il est vrai que le recul n'est que de cinq mois. Ainsi, un suivi prolongé de cette enfant pourrait apporter des informations précieuses concernant l'évolution de cette entité rare après traitement chirurgical. 
Les auteurs déclarent ne pas avoir de lien d'intérêts.

\section{Références bibliographiques}

1. Morgan T, Anderson J, Jorden M, Keller K, Robinson T, Hintz S. Pulmonary glial heterotopia in a monoamniotic twin. Pediatr Pulmonol. 2003;36:162-6.

2. Dettmer P, Beck M, Eufinger H, Rossler L, Tannapfel A, Coerdt W, et al. Bilateral cystic pulmonary glial heterotopia and palatinal teratoma causing respiratory distress in an infant. J Pediatr Surg. 2009;44:2206-10.

3. Rademaker MV, Bakker PM, Bots GTAM, Endtz LJ. Heterotopic nervous tissue in the lungs. Neuropediatrics. 1980;11:284-90.

4. Kershisnik MM, Kaplan C, Craven CM, Carey JC, Townsend JJ, Knisely AS. Intrapulmonary neuroglial heterotopia. Arch Pathol Lab Med. 1992;116:1043-6.

5. Kanbour AI, Barmada MA, Klionsky B, Moossy J. Anencephaly and heterotopic central nervous tissue in lungs. Arch Pathol Lab Med. 1979;103:116-8.

6. Rizzuti T, Ferrarese S, Varesi G, Masini T. Ectopic neuroglial tissue associated with intrapulmonary congenital cystic adenomatoid malformation. Minerva Pediatr. 1997;49:89-92.

7. Gonzalez-Crussi F, Boggs JD, Raffensperger JG. Brain Heterotopia in the Lungs: A Rare Cause of Respiratory Distress in the Newborn. Am J Clin Pathol. 1980;73:281-5.

8. Quemelo PRV, Sbragia L, Peres LC. Development of an experimental model of brain tissue heterotopia in the lung. Int J Exp Pathol. 2007;88:337-42.

9. Alonso L, Sevilla J, Gonzalez-Vicent M, Abad L, Gonzalez-Mediero I, Diaz MA. Pulmonary glial heterotopia in a child diagnosed with fanconi anemia and epilepsy. J Pediatr Hematol Oncol. 2011;33:462-4.

10. Peres LC, Barbosa GHT de S. Brain tissue aspiration neural tube defect. Arq Neuropsiquiatr. 2005;63:614-7. 


\section{Légendes :}

Figure 1. Radiographie thoracique montrant de volumineuses lésions bulleuses (*) de l'hémichamp pulmonaire droit (A). Confirmation scannographique de la présence de plusieurs lésions kystiques $(+)(\mathrm{B}, \mathrm{C}) \mathrm{IRM}$ anténatale montrant plusieurs lésions hétérogènes en discret hyposignal T2 (>) (D)

Figure 1. Chest X-rays showing large radioluscent blebs $\left(^{*}\right)$ mostly located in right lung (A) Scanning confirmation of multiple cystic lesions (+) $(\mathrm{B}, \mathrm{C})$ Antenatal MRI showing several herogenous lesions presenting a subtle hyposignal in T2 weighting (>) (C)

Figure 2. Pièce de lobectomie inférieure droite montrant une lésions kystique ouverte en deux mesurant $4,5 \mathrm{~cm}$ de diamètre $(*)$.

Figure 2. Right lower pulmonary lobectomy showing open cystic lesion measuring $4,5 \mathrm{~cm}$ in diameter $(*)$.

Figure 3. Coloration HE (x10): Lésions kystiques disséminées au sein du parenchyme pulmonaire à faible grandissement (* : lumière du kyste) (A). Coloration HE (x30) : paroi des kystes constituée de tissu glial peu cellulaire agencé en foyer vaguement nodulaire $(+)$ avec un épithélium bronchique en surface (<) (B). Coloration HE (x200) : lésion exclusivement composée de tissu glial bien différentié (**) (C). Etude immunohistochimique avec l'anticorps PS100 (x40) : expression cytoplasmique hétérogène de la PS100 par le tissu glial $(++)(\mathrm{D})$.

Figure 3. HE staining (x10) : cystic lesions disseminated within the lung parenchyma at low magnification (* : lumen of the cyst) (A). HE staining (x30) : cyst walls composed of slightly cellular glial tissue arranged in nodular pattern $(+)$ and lined with bronchial epithelium $(<)$ (B) . HE staining (x200) : lesion exclusively composed of well differentiated glial tissue $(* *)$ (C) Immunohistochemistry using PS100 antibody (x40) : Heterogeneous expression of PS100 by glial tissue $(++)(\mathrm{D})$. 


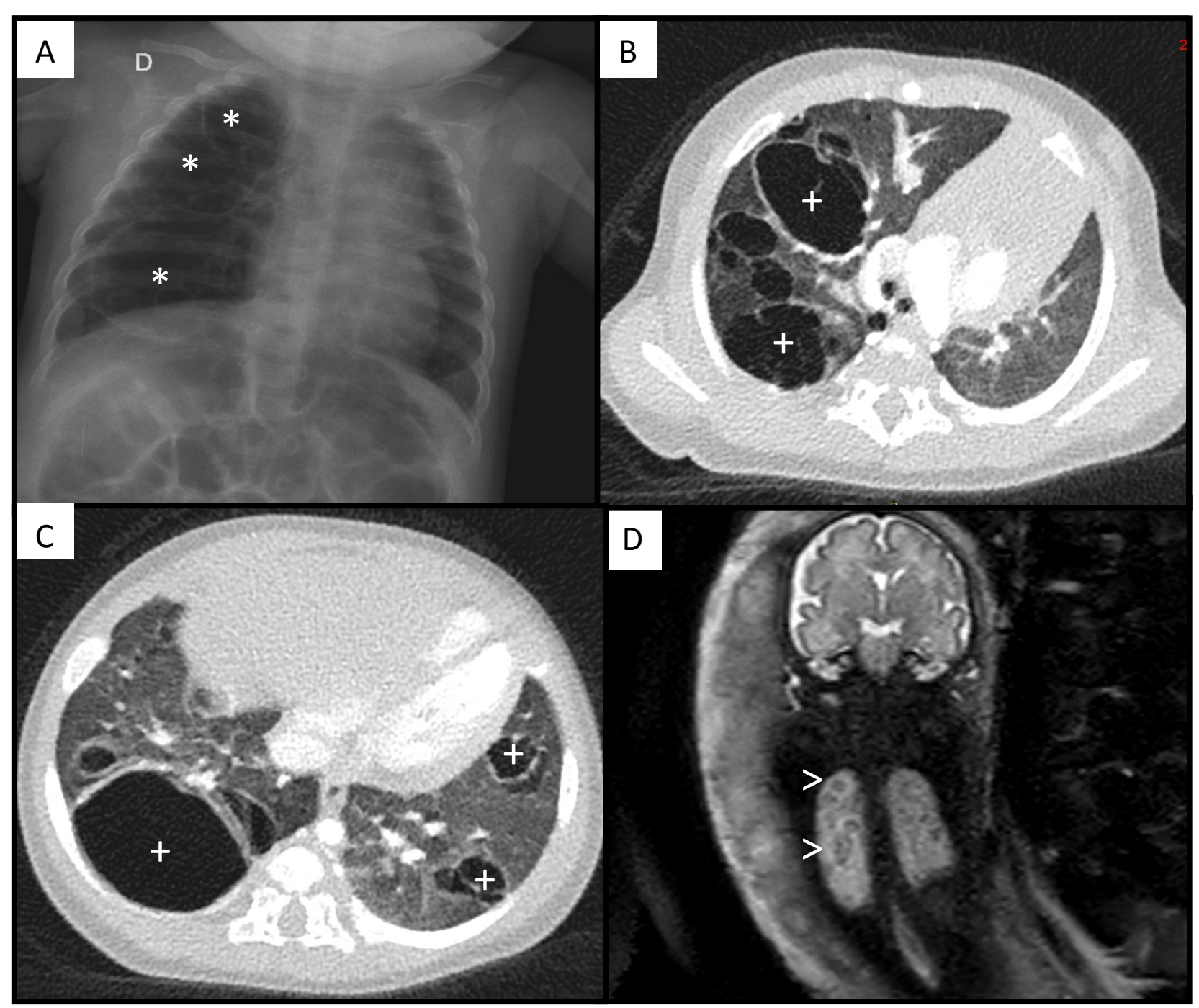




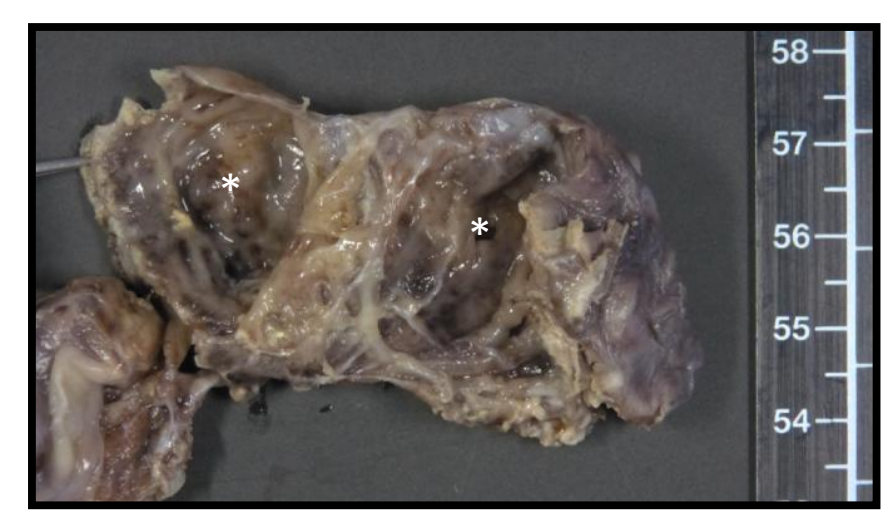




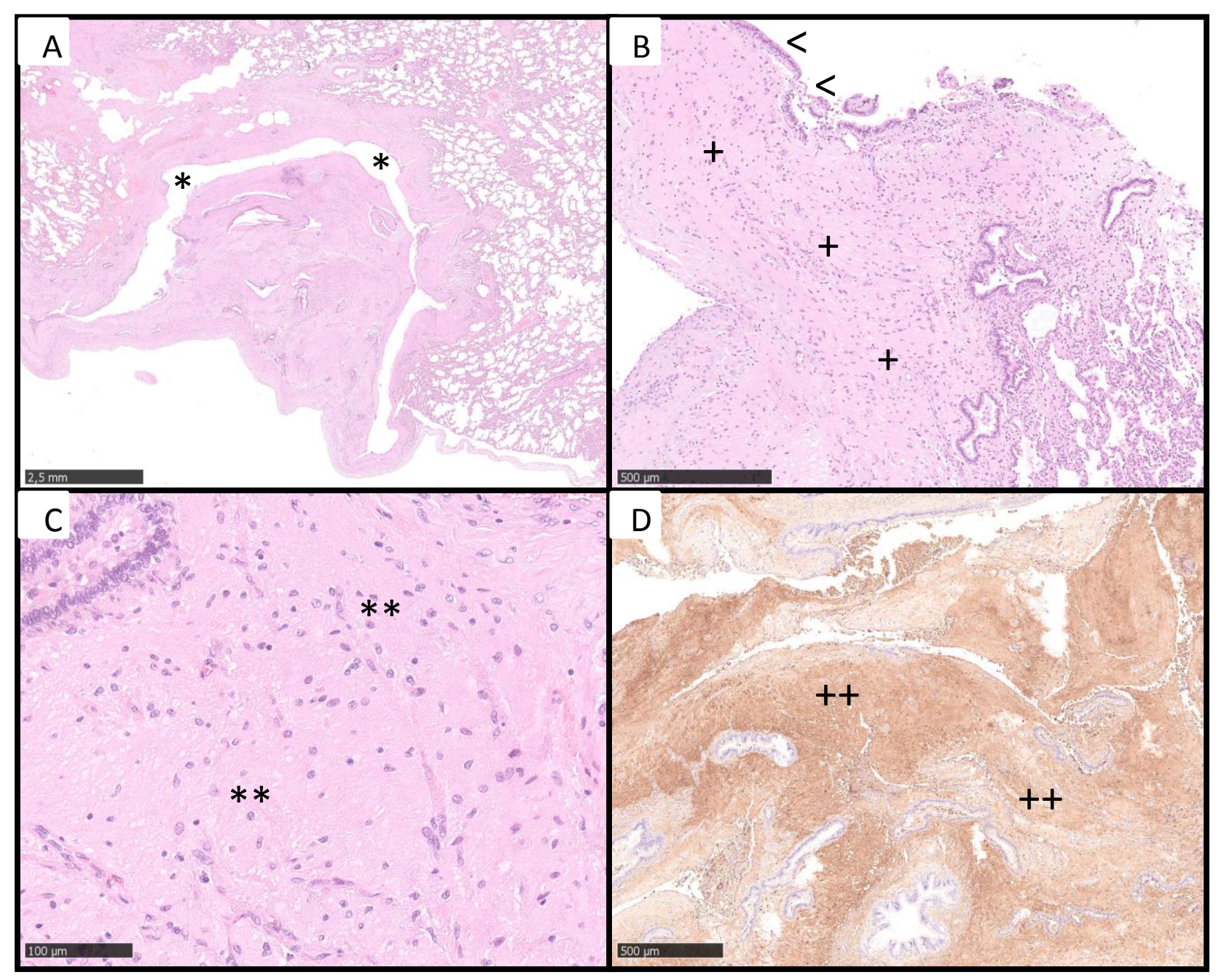

